\title{
HIDDEN MOMENTUM AND THE FORCE ON A MAGNETIC DIPOLE
}

\author{
V. HNIZDO \\ Department of Physics and Schonland Research Centre for Nuclear Sciences \\ University of the Witwatersrand, Johannesburg, 2050 South Africa
}

(Received February 24, 1992)

\begin{abstract}
The problem of the correct expression for the force on a magnetic dipole in vacuum is discussed. Different models of magnetic dipole are considered and particular attention is given to the presence of hidden momentum, which modifies the usual expression for the force on a current-loop dipole when the external electromagnetic field is not static.
\end{abstract}

\section{INTRODUCTION}

Classical electrodynamics is, together with classical mechanics, a cornerstone on which the imposing edifice of the present-day physics, and thus much of modern technology, is built. It may therefore come to many as a surprise that, in this wellestablished discipline, such a basic and practical problem as that of the expression for the force on a magnetic dipole in vacuum has been the subject of ongoing discussions and controversy for many years. A proper understanding of this problem with its unexpectedly subtle aspects has been gained only relatively recently, after the realization 25 years ago that a current-carrying body whose centre of mass is at rest can nevertheless have a nonzero linear momentum, called hidden momentum $[1,2]$. It is felt that these findings, which have been so far confined to the literature on pure physics, would be of interest to a wider audience, in particular to one concerned with technological applications utilizing magnetic forces on matter.

The present paper is an account of the problem of the force on a magnetic dipole in vacuum, and its solution in the current physics literature on electromagnetism. The paper is written from a physics, as opposed to technology perspective, but the problem is treated informally using physical arguments only, with references given to more rigorous derivations and calculations. In the second section of the paper, the magnetic-charge and current-loop models of magnetic dipole are introduced and their differences and relevance to physics are discussed. In the third section, the concept and necessity of hidden momentum are explained. In the last section, the correct expression for the force on a current-loop magnetic dipole, which takes into account the existence of hidden momentum, is derived. 


\section{DIFFERENT MODELS OF MAGNETIC DIPOLE}

The magnetic dipole is the simplest system that produces a magnetic field. In vacuum, it is, apart from its mass, specified completely by giving its magnetic dipole moment, which is the lowest-order magnetic moment as no free magnetic charges, or monopoles, are found in nature. Nevertheless, in analogy with electrostatics, it is sometimes found useful to model a magnetic dipole as two opposite magnetic charges separated by a small distance. The magnetic field produced by such a magneticcharge dipole is the same as that of a dipole that is modelled by an electric current loop, i.e., a dipole whose moment is that of a localized electric current distribution. We assume the limit of a point magnetic dipole, or, perhaps more physically, that the dimensions of the dipole are sufficiently small compared to the distances at which its magnetic field would be considered. This assumption is, of course, already implied by our disregard of magnetic moments in the dipole that are of higher order than magnetic dipole; such moments, strictly speaking, do not vanish in systems of finite dimensions.

The two models of magnetic dipole lead, however, to different expressions for the force that the magnetic dipole experiences in an external electromagnetic field. The expression for the force on a magnetic-charge dipole is analogous to that for an electric dipole, and is given by [3]

$$
\mathbf{F}=(\mathbf{m} \cdot \nabla) \mathbf{B}-\mu_{0} \varepsilon_{0} \frac{d \mathbf{m}}{d t} \times \mathbf{E} .
$$

Here $\mathbf{m}$ is the magnetic dipole moment of the dipole, $\mathbf{B}$ is an external magnetic flux density and $\mathbf{E}$ is an external electric field. To free the discussion from the problems with defining the magnetic dipole moment of a body that is immersed in a permeable medium [4], we shall consider here only the case when $\mathbf{B}=\mu_{0} \mathbf{H}$, i.e., when the permeability of the external medium equals that of the vacuum. Also, it will be always assumed that the dipole is at rest to avoid complications connected with the fact that a moving magnetic dipole has an effective electric dipole moment, in addition to the magnetic one. It is important to note that we do not assume that the external fields $\mathbf{B}$ and $\mathbf{E}$ have to be static and that even the magnetic moment $\mathbf{m}$ can vary with time, giving rise to the second term in Eq. (1).

On the other hand, the force on a current-loop magnetic dipole, as for example derived in the authoritative textbook of Jackson [5], is given as

$$
\mathbf{F}=\nabla(\mathbf{m} \cdot \mathbf{B})
$$

To see the difference between the forces of Eqs. (1) and (2) more clearly, one can use in Eq. (2) the vector identity $\nabla(\mathbf{m} \cdot \mathbf{B})=(\mathbf{m} \cdot \nabla) \mathbf{B}+\mathbf{m} \times(\nabla \times \mathbf{B})$ and Maxwell's equation

$$
\nabla \times \mathbf{B}=\mu_{0} \mathbf{J}+\mu_{0} \varepsilon_{0} \frac{\partial \mathbf{E}}{\partial t},
$$


to get

$$
\mathbf{F}=(\mathbf{m} \cdot \nabla) \mathbf{B}+\mu_{0} \mathbf{m} \times \mathbf{J}+\mu_{0} \varepsilon_{0} \mathbf{m} \times \frac{\partial \mathbf{E}}{\partial t} .
$$

Thus, in the static case when both $d \mathrm{~m} / d t$ and $\partial \mathrm{E} / \partial t$ vanish, the magnetic-charge and current-loop expressions for the force on a magnetic dipole, Eqs. (1) and (4), respectively, differ only when the dipole is located at a point where the external current density $\mathbf{J}$ is not zero.

We know from Maxwell's equations that magnetic fields are produced by electric currents, and not by the fictitious magnetic charges so beloved by old textbooks on magnetostatics. But what about the magnetic moments of electrons and atomic nuclei, whose existence is intimately connected with that of the spin angular momentum and thus as such is outside the domain of classical electrodynamics? Is the force on the magnetic moment of an elementary particle given correctly by the magnetic-charge model, or by the current-loop model? Materials with a nonzero magnetization $\mathbf{M}$ carry an equivalent current density $\mathbf{J}=\nabla \times \mathbf{M}$, and the study of the scattering of particles with magnetic moment from such materials should provide an answer to this question. Experiments with the scattering of polarized neutrons from ferromagnetic materials in the early 1950's [6] showed conclusively that the force on the magnetic dipole moment of the neutron has the term $\mu_{0} \mathbf{m} \times \mathbf{J}$ predicted by the current-loop model in Eq. (4). It is now generally accepted that the magnetic moments of electrons and atomic nuclei can be modelled accurately by circulating electric currents.

The current-loop model is then the physically correct representation of all magnetic dipoles occurring in nature, including those that arise from a magnetization, permanent or induced, of material bodies. For a finite-size body of magnetizable matter placed in an external magnetic field, the expression for the force on a magnetic dipole must be suitably adapted to reflect the fact that the magnetic moment of such a body depends on the value of the field inside the body and that this field is different from the external field. We shall not be concerned here with these wellknown facts, important they may be in many applications, and shall concentrate on the investigation of the relevance of hidden momentum for the force on a magnetic dipole. It will turn out, surprisingly, that Eq. (4) (or, equivalently, Eq. (2)) does not give the force on a current-loop magnetic dipole entirely correctly when the external electromagnetic field, or the magnetic dipole moment itself, varies with time.

\section{HIDDEN MOMENTUM}

Let us consider a system consisting of a magnetic dipole and a charged particle, with the magnetic dipole being at rest and the charge moving at the given instant of time. The magnetic field of the magnetic dipole acts on the moving charge, whose motion creates a magnetic field that acts in turn on the magnetic dipole. With the 
force on the magnetic dipole as given by Eq. (2) (or, equivalently, Eq. (4), where the external current density $\mathbf{J}$ is now, of course, zero), one can show easily that Newton's third law is violated for the forces acting between the magnetic dipole and the charge. This would mean that the momentum associated with the motion of a charge and a current-loop magnetic dipole is not conserved in their interaction. This conclusion can be reached more directly by considering the magnetic dipole and charge initially both at rest, but with the magnitude of the dipole moment of the dipole starting to decrease gradually [1] (for example, this may be accomplished with a superconducting-loop dipole that is gradually being warmed up). According to Faraday's law, the time variation of the magnetic field of the dipole generates an electric field, which imparts an impulse to the charge. According to Eq. (2), there is, however, no reaction force that the initially stationary charge could exert back on the magnetic dipole, and thus the centre of mass of the closed system of the dipole and charge would start to move. To put it figuratively, the system would be lifting itself by its bootstraps.

While the above conclusion is paradoxical, there would be in fact no conflict with the conservation of the total (i.e., mechanical plus electrodynamic) momentum in a closed system. Even for a static configuration of a magnetic dipole and charge, the combined magnetic flux density $\mathbf{B}$ of the dipole and the electric field $\mathbf{E}$ of the charge contain a nonzero momentum, given by [7]

$$
\mathbf{p}_{\text {field }}=\varepsilon_{0} \int \mathbf{E} \times \mathbf{B} d^{3} r=-\mu_{0} \varepsilon_{0} \mathbf{m} \times \mathbf{E},
$$

where $\mathbf{E}$ on the right-hand side is the electric field of the charge at the magnetic dipole $\mathbf{m}$. It is from this electromagnetic field momentum that the momentum of the motion of the charge would be drawn in the above example. In general, only the sum of the field momentum plus mechanical momentum must be conserved. Do we then have to accept that "bootstrap spaceships" are possible? It should come to us as a relief (at least from the physics point of view) that the answer is no, and the reason is as follows. Using only the most general assumptions, one can show that the total momentum of a closed system of any stationary distribution of charge and current is zero $[8,9]$. As the momentum of the electromagnetic field in such a system does not generally vanish, this means that there must be an equal and opposite momentum "hidden" in the matter carrying the stationary distribution of charge and current. Thus a magnetic dipole in the electric field of a charge contains in general a hidden momentum that is not associated with its translational motion, and this hidden momentum is equal and opposite to the field momentum of Eq. (5). The existence of the hidden momentum must be taken into account when considering the force on a magnetic dipole, and then it turns out that Newton's third law is satisfied for the interaction of the dipole with the charge.

Before we come finally to the correct expression for the force on a current-loop magnetic dipole, let us try to understand in qualitative terms the surprising and 
counterintuitive result that a stationary body can have a nonzero linear momentum. Here we must consider specific models of the current-loop dipole. Broadly speaking, there are three classes of such dipoles, which can be described schematically as follows [7]: (i) a gas of charged particles that circulate inside an insulating tube, (ii) a charged incompressible fluid moving inside an insulating tube, and (iii) a current loop in a conducting body. In model (i), the positively charged particles move faster in the parts of the tube where the external electric field has a component along the direction of the motion of the particles, and they move slower where the external electric field is directed against their motion; the effect of the external electric field on the motion of the negatively charged particles in the tube is exactly opposite. The changes in the speed of the charged particles lead to changes in their relativistic mass, and when these effects are integrated along the closed path of these particles, a net nonzero momentum $\mu_{0} \varepsilon_{0} \mathbf{m} \times \mathbf{E}$ is obtained [7, 10]. This hidden momentum is exactly equal and opposite to the electromagnetic field momentum, Eq. (5), in the system. In model (ii), the velocity of the charged incompressible fluid in the tube cannot change in response to the external electric field, but this field induces a stress in the local rest frame of the fluid. The relativistic transformation of the stress-energy tensor to the rest frame of the tube yields a nonzero momentum density along the tube, which gives on integration exactly the same hidden momentum as model (i) [7].

We have seen that the hidden momentum in models (i) and (ii) is due to relativistic effects in the internal motion of the current carriers and relativistic properties of the mechanical stress, respectively. It should be understood that these effects, unlike usual relativistic effects, are completely independent of the velocities involved, which are here the velocities of the current carriers. Model (iii) is different from the other models due to the fact that here the hidden momentum vanishes because the external electric field is screened off by the conductor and thus it cannot act on the current carriers. This means that the electromagnetic field momentum must be zero in this case too, which fact can be shown to follow from the circumstance that the net electric potential is constant in the body of a conductor [7, 8]. In model (iii), however, the current in the current loop interacts with the current of the charges induced in the conductor by a time-varying external electric field, and one can show that this interaction leads to a term in the force on the magnetic dipole that is exactly the same as if the dipole had the hidden momentum of models (i) and (ii) [11]. The three different models should cover, in a schematic fashion, all the possible cases of magnetic dipoles generated by electric currents.

\section{FORCE ON A CURRENT-LOOP MAGNETIC DIPOLE}

We now return to the question of the correct expression for the force on a currentloop magnetic dipole. Due to the presence of hidden momentum in the body that carries the magnetic dipole moment (in model (iii) of magnetic dipole, one should 
speak properly about an effective hidden momentum), the expression of Eq. (2) actually gives only the rate of change of the total momentum of the dipole

$$
\nabla(\mathbf{m} \cdot \mathbf{B})=\frac{d}{d t}\left(m \mathbf{v}+\mu_{0} \varepsilon_{0} \mathbf{m} \times \mathbf{E}\right)
$$

which is the sum of the momentum $m v$ of its translational motion and its hidden momentum $\mu_{0} \varepsilon_{0} \mathbf{m} \times \mathbf{E}$. The force that determines the acceleration of the dipole, i.e. $m d \mathbf{v} / d t$, is then given correctly as [2]

$$
\mathbf{F}=\nabla(\mathbf{m} \cdot \mathbf{B})-\mu_{0} \varepsilon_{0} \frac{d}{d t}(\mathbf{m} \times \mathbf{E}) .
$$

Using Eq. (4) instead of Eq. (2), we get for this force an alternative expression

$$
\mathbf{F}=(\mathbf{m} \cdot \nabla) \mathbf{B}+\mu_{0} \mathbf{m} \times \mathbf{J}-\mu_{0} \varepsilon_{0} \frac{d \mathbf{m}}{d t} \times \mathbf{E},
$$

which, on comparison with Eq. (1), shows that the correct expression for the force on a current-loop dipole differs from that on a magnetic-charge dipole only by the term $\mu_{0} \mathbf{m} \times \mathbf{J}$. It should be stressed that, unless $d \mathbf{m} / d t$ does not vanish, there is a difference between the force on a current-loop dipole as given by Eq. (4) on the one hand and the correct expression of Eq. (8) on the other only when the external electric field varies with time. This difference should be observable in a suitable time-varying electromagnetic field.

It is not difficult to demonstrate that with the correct force on a current-loop dipole, Eq. ( 7) or (8), Newton's third law holds for the interaction of the magnetic dipole with a charged particle, even in the general case when the magnetic moment $\mathbf{m}$ varies with time [12]. In fact, a general conservation statement holds that asserts that the mechanical momentum associated with the motion of current-carrying bodies and charged particles in a closed system is conserved itself in the nonrelativistic limit, independently of the electromagnetic field momentum in the system [10]. In this connection, it is interesting to note that angular momentum is different in this respect, as no part of the mechanical angular momentum is conserved alone, and only the sum of electromagnetic field angular momentum plus mechanical angular momentum, which in general contains a hidden part, is a conserved quantity in a closed system [12].

\section{REFERENCES}

1. W. Shockley and R.P. James, Phys. Rev. Lett., 18, 876 (1967).

2. H.A. Haus and P. Penfield, Phys. Lett., 26A, 412 (1968).

3. B.D.H. Tellegen, Am. J. Phys., 30, 650 (1962).

4. F.J. Lowes, Proc. R. Soc. Lond. A, 337, 555 (1974).

5. J.D. Jackson, Classical Electrodynamics, (Wiley, New York, 1975), 2nd ed., pp. $180-186$.

6. D.J. Hughes and M.T. Burgy, Phys. Rev., 81, 498 (1951). 
7. L. Vaidman, Am. J. Phys., 58, 978 (1990).

8. M.G. Calkin, Am. J. Phys., 39, 513 (1970).

9. Y. Aharanov, P. Pearle and L. Vaidman, Phys. Rev. A, 37, 4052 (1988).

10. W.E. Furry, Am. J. Phys., 37, 621 (1969).

11. V. Hnizdo, Am. J. Phys., 60, 279 (1992).

12. V. Hnizdo, Am. J. Phys., 60, 242 (1992).

Vladimir Hnizdo obtained his M.Sc. degree in physics from the Technical University of Prague, Czechoslovakia in 1966, and his Ph.D. degree in nuclear physics from the University of Birmingham, England in 1971. He has been at the University of the Witwatersrand, Johannesburg since 1974. His research interests are primarily in nuclear physics.

Keywords: classical electrodynamics, magnetic dipole, hidden momentum, momentum conservation, current-loop dipole, magnetic-charge dipole. 